SMALL INTESTINE

\title{
A novel method for rapidly diagnosing the causes of diarrhoea
}

\author{
C S J Probert, P R H Jones, N M Ratcliffe
}

Gut 2004;53:58-61

See end of article for authors' affiliations

.....................

Correspondence to:

Dr C S J Probert,

Department of Medicine,

Bristol Royal Infirmary,

Marlborough St, Bristol

BS2 8HW, UK;

c.s.j.probert@bristol.ac.uk

Accepted for publication 8 July 2003
Background: The microbiological diagnosis of infectious diarrhoea may take several days using conventional techniques. In order to determine whether flatus can be used to make a rapid diagnosis, the volatile organic compounds associated with diarrhoea were analysed.

Methods: Stool samples were collected from 35 patients with infectious diarrhoea and from six healthy controls. Gaseous compounds were extracted from a headspace using solid phase microextraction and analysed using gas chromatography and mass spectroscopy.

Results: Characteristic patterns of volatile gases were found for the main causes of infectious diarrhoea in hospitals. Furan species without indoles indicated Clostridium difficile, ethyl dodecanoate indicated rotavirus, ammonia without ethyl dodecanoate suggested other enteric viruses, and the absence of hydrocarbons and terpenes indicated Campylobacter infection.

Conclusion: These results could be the basis of rapid near patient diagnosis of infectious diarrhoea.
$\mathrm{D}$ iarrhoea due to infection is a major cause of morbidity and mortality. In England and Wales, there are approximately 15000 cases of Clostridium difficile infection per annum (www.PHLS.co.uk). It is estimated that in an average sized district general hospital, 100 cases of $C$ difficile infection can be expected each year with an extra annual cost of $£ 400000$ and 2100 lost bed days. ${ }^{1}$ This is equivalent to £60M for England and Wales as a whole. In the USA, rotavirus infection accounts for $48 \%$ of viral gastroenteritis and 56000 hospitalisations per year, ${ }^{2}$ while in developing countries 600000 children die from rotavirus each year. ${ }^{3}$

Despite the development of enzyme linked immunosorbent assays (ELISAs) and molecular techniques to determine the aetiology of infectious diarrhoea, in most cases there is a delay of several days between the collection of a stool sample and a microbiological diagnosis. Indeed, a delay of 8.2 days has been reported recently. ${ }^{4}$ The delay is, in part, due to shipping of the samples to an appropriate laboratory but mainly due to the time required to complete the diagnostic techniques in common use. The limited availability of expensive microbiological techniques in developing countries compounds the problem. In some epidemics, stool samples have been flown to the USA for microbiological processing. Depending on the patient's circumstances, the results of a diagnostic delay is likely to range from lost productivity to prolonged hospitalisation or death. There is a pressing need to make a rapid accurate diagnosis in all patients.

Diarrhoea has a wide variety of physical characteristics that have been used to try to determine its aetiology, with limited success. The vapour that accompanies diarrhoea has not been used for diagnostic purposes, nor has the characteristics of these volatile organic compounds (VOCs) received any detailed attention.

To test the hypothesis that VOCs exist which are specific to the cause of diarrhoea, a study of stool samples from patients with various causes of diarrhoea and from healthy volunteers was undertaken.

\section{MATERIALS AND METHODS Patients}

Stool samples were obtained from 38 subjects with diarrhoea and from six healthy controls. For 35 subjects, the aetiology of the diarrhoea had been previously determined by routine microbiological assessment. The samples were a mixture of fresh stool and frozen archived samples. The cause of diarrhoea was known for each but no other clinical data were recorded. Of these samples, diarrhoea was due to $C$ difficile $(\mathrm{n}=6)$, Campylobacter $(\mathrm{n}=5)$, rotavirus $(\mathrm{n}=5)$, small rounded structured virus (SRSV, $\mathrm{n}=9$ ), adenovirus $(n=5)$, astrovirus $(n=2)$, and giardiasis $(n=3)$.

Three of the subjects had a clinically diagnosed exacerbation of inflammatory bowel disease involving the colon without microbiological assessment. No specimens had been sent for microbiological testing although none had received antibiotics and each responded to treatment for the relapse.

The healthy controls (aged 40-65 years; three males) each provided a stool sample the form of which was normal for them; none had gastrointestinal symptoms and none had previously been diagnosed with a chronic gastrointestinal disorder.

In addition, samples were obtained from nine subjects (aged 0.5-49 years; two females) who developed diarrhoea following bone marrow transplantation (BMT). Each had received prophylactic broad spectrum antibiotics. Full microbiological assessment for common bacterial and viral causes for diarrhoea were performed as well as ELISA for $C$ difficile toxin. No bacterial pathogens were identified. These samples were used as controls to compare with those from patients with $C$ difficile. However, as the aetiology of post BMT diarrhoea is often unexplained, these samples were excluded from the remainder of the study and not used in the generation of summary statistics.

\section{Analysis}

Stool samples (approximately $0.75 \mathrm{~g}$ ) were portioned in $10 \mathrm{ml}$ sealed headspace vials (Supelco) designed for solid phase microextraction (SPME). Gas within the headspace

\footnotetext{
Abbreviations: ELISA, enzyme linked immunosorbent assay; VOCs, volatile organic compounds; SRSV, small rounded structured virus; BMT, bone marrow transplantation; SPME, solid phase microextraction; GC, gas chromatography; MS, mass spectroscopy; PPV, positive predictive value; NPV, negative predictive value
} 
was then sampled and analysed using gas chromatography (GC) and mass spectroscopy (MS). The technique used was based on that which we have employed to identify diseased vegetables. $^{56}$

Samples were equilibrated in a water bath $\left(37^{\circ} \mathrm{C}\right)$ and their volatile compounds adsorbed from the headspace onto the SPME fibre (Supelco, $85 \mu \mathrm{m}$ carboxen/polydimethylsiloxane). Any samples not extracted within one hour of collection were stored in vials at $-18^{\circ} \mathrm{C}$. Sample volatile compounds were desorbed from the fibre thermally via splitless injection (HP 5890 II, 1.5 minutes, $250^{\circ} \mathrm{C}$ ) onto a thick film column (Supelco, SPB-1 Sulphur, $30 \mathrm{M} \times 0.32 \mathrm{~mm}, \beta 20$ ). Volatile compounds were eluted from the column $\left(35^{\circ} \mathrm{C}\right.$ ( 5 minutes $) \times 10^{\circ} \mathrm{C} /$ minute $-250^{\circ} \mathrm{C}(2$ minutes $), 1 \mathrm{ml} / \mathrm{min}$ ) directly into a quadruple mass spectrometer (HP 5971) full scan (positive mode, 15-60 amu, 2 minutes, 19-350 amu until 28.5 minutes). Compounds were identified by spectral analysis undertaken using proprietary Hewlett Packard software (G1034C, V1.05) running the NBS75K library, and also by their elution order.

\section{Statistics}

The proportion of samples, from each group of patients, that exhibited a particular characteristic were compared with the proportion of the remainder exhibiting the same characteristic using the $\chi^{2}$ test with Yates' correction. Positive and negative predictive values, and sensitivity and specificity were also calculated based on these proportions.

\section{RESULTS}

VOCs were successfully adsorbed onto short $1 \mathrm{~cm}$ fibres using a recently developed SPME technique. Thermal desorption and analysis were undertaken via a small bench top GC-MS system. The composition of VOCs of stools from normal subjects were remarkably similar (fig lA shows a typical example) but among patients with various types of diarrhoea, the distribution of VOCs was consistently different. Characteristic changes in the volatile pattern occurred with specific types of diarrhoea (fig $1 \mathrm{~B}$ shows a typical example from a patient with $C$ difficile).

\section{Normal subjects}

Analyses of the volatiles emitted from the stool of normal subjects $(n=6)$ showed that the predominant class of volatile was the phenols, which accounted for $20-50 \%$ of the total volatiles (fig 2), 4-methylphenol being by far the most prevalent. Indole and 3-methylindole were also abundant (5-25\%) in normal subjects, as were a group of volatiles classified, without detailed differentiation, as terpenes and hydrocarbons (10-20\%). A range of organic acids were also present but acids as a class of compounds were present in all the samples and therefore cannot be considered as marker compounds for diarrhoea. The principle volatile components of normal flatus were thus 4-methylphenol, indole, 3-methylindole, and limonene. Benzaldehyde was ubiquitous although less abundant.

Indoles were present in most subjects with diarrhoea (fig 2). Their occurrence is related to the metabolism of tryptophan by Escherichia coli. Indeed, a simple laboratory test for $E$ coli based on the detection of indole has been in clinical use since $1963 .^{7}$

\section{Clostridium difficile}

In flatus from patients $(n=6)$ with diarrhoea due to $C$ difficile, furans (principally 2-furancarboxaldehyde and 5-methyl-2-furancarboxaldehyde) were particularly prevalent (25-55\% of the measured volatiles) (fig 2). 3-Methylindole was present in a minority of patients with $C$ difficile (1/6). Stool samples from patients with diarrhoea following BMT
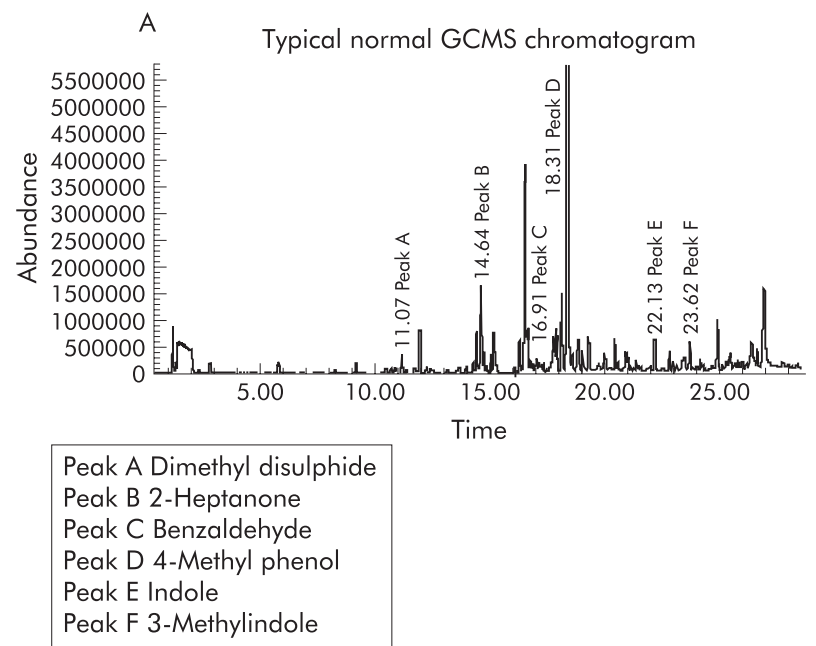

B Typical Clostridium difficile GCMS chromatogram

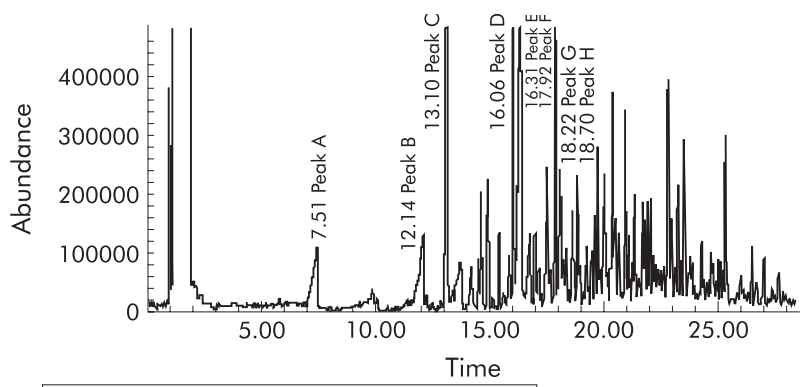

\begin{tabular}{|l|}
\hline Peak A Acetic acid \\
Peak B Butanoic acid \\
Peak C 2-Furancarboxaldehyde \\
Peak D 5-Methyl-2-furancarboxaldehyde \\
Peak E Methyl furancarboxylate \\
Peak F 2-Hydoxy benzaldehyde \\
Peak G 4-Methyl phenol \\
Peak H 2-Methoxy phenol \\
\hline
\end{tabular}

Figure 1 (A) Typical example of a gas chromatography-mass spectroscopy (GCMS) chromatogram in a normal subject. (B) Typical example of a GCMS chromatogram in a patient with Clostridium difficile.

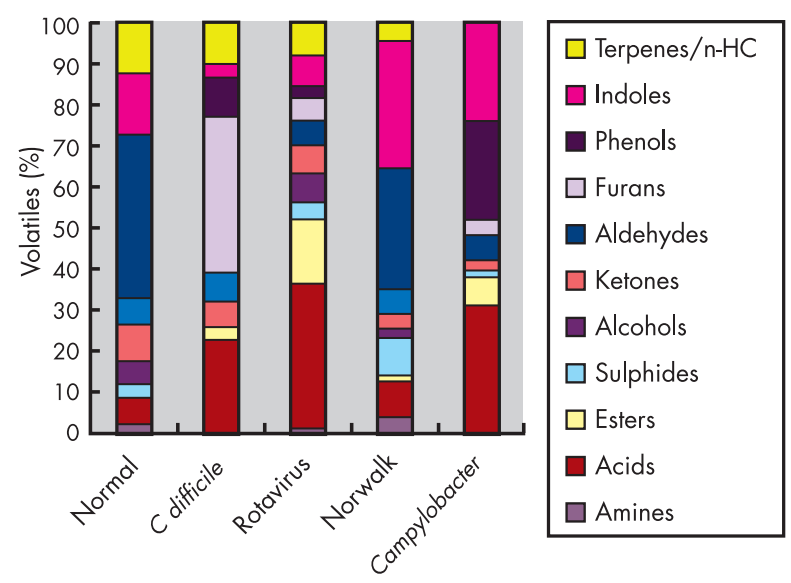

Figure 2 Metabolic profile of volatile classes from stool samples of normal subjects and from patients with infectious diarrhoeas (typically an average of six samples for each sample type). 
were also unlikely to contain 3-methylindole (0/9). High levels of 5-methyl-2-furancarboxaldehyde were found in 5/6 $C$ difficile patients but only in one of the remaining 38 samples of patients with infectious diarrhoea and exacerbation of inflammatory bowel disease. Used alone the presence of 5-methyl-2-furancarboxaldehyde was significantly associated with $C$ difficile infection $\left(\chi^{2}=22.2\right.$, $\mathrm{p}=0.000002$ ). Furthermore, 5-methyl-2-furancarboxaldehyde without measurable 3-methylindole occurred exclusively among patients with $C$ difficile $\left(\chi^{2}=20.4\right.$, $\mathrm{p}=0.000002)$. This combination had a positive predictive value (PPV) of $100 \%$ but two cases of $C$ difficile would have been overlooked, lowering the sensitivity to $66 \%$ and the negative predictive value (NPV) to $95 \%$. In contrast, 2furancarboxaldehyde without measurable 3-methylindole was found in 5/6 cases with two "false positive" cases among the remainder $\left(\chi^{2}=22.2, \mathrm{p}=0.000003\right.$, sensitivity $=83 \%$, specificity $=97 \%$, PPV $83 \%$, and NPV 97\%). All of these associations and their respective sensitivity and specificities, and PPV and NPV are summarised in table 1 .

None of the nine samples of diarrhoea following antibiotics associated with BMT contained furans however 3-methyl indole was suppressed. These samples were not used for statistical analysis although to have done so would have strengthened the argument regarding $C$ difficile.

\section{Rotavirus}

It is a striking observation that ethyl dodecanoate was ubiquitous in the VOCs of stools from patients with rotavirus $(n=5)$. Only one other sample, from a patient with enteritis due to adenovirus infection, contained this and we cannot exclude the possibility of dual pathology. It is also of interest that dodecanoic acid was detected in four of five of the rotavirus samples and absent from all but one of the remaining 39 samples analysed. Propyl dodecanoate was not detected in any of the samples analysed except for one patient with rotavirus. Thus ethyl dodecanoate was a very sensitive (100\%) and specific (97\%) marker for rotavirus (table 1). Ethanol was also ubiquitous with rotavirus but it was also present in the flatus from two of the normal subjects and six patients with diarrhoea. The presence of ethanol in many beverages precludes its use as a marker compound, except in specific circumstances.

\section{Norwalk virus and other enteric infections}

Patients with diarrhoea due to SRSV2 (often called the Norwalk virus) $(n=9)$ were studied. Ammonia was found in seven of the samples but it is not known whether all nine subjects had the same strain of virus. Furthermore, one sample yielded very few volatiles and was the smallest volume of stool in the study. Ammonia was also found in both subjects with astrovirus, two of five with adenovirus, one of three with giardiasis, and one of five with rotavirus. Therefore, ammonia on its own cannot be used to diagnose the Norwalk virus although we have never detected it among patients with a bacterial infection and so it may have a role in the diagnosis of viral enteritis. Indeed, ammonia was found in $12 / 19$ cases of non-rotavirus enteritis and giardiasis compared with just one, a rotavirus case, of the remaining 25 samples $\left(\chi^{2}=15.4, \mathrm{p}<0.0001\right)$. Ammonia is a difficult gas to detect and volatility depends to a high degree on the $\mathrm{pH}$ of the sample. It may be that some sample preconditioning is required for an ammonia assay to be of significant diagnostic value.

Ammonia without ethyl dodecanoate was found in 11/19 cases of non-rotavirus enteritis but not in any other sample $\left(\chi^{2}=16.3, p=0.00005\right)$. This combination has $100 \%$ specificity and 58\% sensitivity for non-rotavirus viral enteritis.

\section{Campylobacter jejuni}

Among patients with Campylobacter $(\mathrm{n}=5)$, phenols were abundant (10-40\% of measured volatiles) as were indoles $(20-30 \%)$. There were also more volatile organic acids (30$35 \%$ of measured volatiles) than in any other group of patients. No VOCs belonging to the terpenes/hydrocarbon groups were found. In contrast, all other samples except three contained terpenes/hydrocarbons $\left(\chi^{2}=19.5, \mathrm{p}=0.00001\right)$. This had $100 \%$ sensitivity and 92\% specificity with an NPV of $100 \%$.

\section{DISCUSSION}

We have combined the recently developed advances in solid phase microextraction with the novel approach of volatile profile analyses from stool samples in order to determine the cause of infectious diarrhoea. Despite the complexity of the VOCs in normal stool, we have found changes in specific diseases.

Furans were prevalent among the VOCs from patients with $C$ difficile infection but furans were not found among the VOCs from normal controls nor in stool samples from the majority of subjects with other forms of infectious diarrhoea. Furans are likely to be related to bacterial metabolism of furanose from dietary fructose. Many Clostridia species are capable of fermenting sugars and amino acids. Efforts have been made to rapidly identify $C$ difficile in cultures by the presence of p-cresol and caproic acid. ${ }^{9}$ However, this

Table 1 Diagnostic value of volatile organic compounds (VOCs) from stool samples from patients with infectious diarrhoea

\begin{tabular}{|c|c|c|c|c|c|c|c|c|c|}
\hline & Marker & $\begin{array}{l}\text { Present in specific } \\
\text { disease }\end{array}$ & $\begin{array}{l}\text { Present in } \\
\text { remainder }\end{array}$ & $\chi^{2}$ & p Value & $\begin{array}{l}\text { Sensitivity } \\
(\%)\end{array}$ & $\begin{array}{l}\text { Specificity } \\
(\%)\end{array}$ & $\begin{array}{l}\text { PPV } \\
(\%)\end{array}$ & $\begin{array}{l}\text { NPV } \\
(\%)\end{array}$ \\
\hline C difficile (i) & $3 \mathrm{Ml}$ negative & $1 / 6$ & $25 / 38$ & 3.3 & 0.06 & 83 & 65 & 28 & 96 \\
\hline C difficile (ii) & 2FC positive & $6 / 6$ & $8 / 38$ & 11.5 & 0.0007 & 100 & 80 & 40 & 100 \\
\hline C difficile (iii) & $\begin{array}{l}3 \mathrm{MI} \text { negative, } \\
2 \mathrm{FC} \text { positive }\end{array}$ & $5 / 6$ & $1 / 38$ & 22.2 & $2.6 \mathrm{E}-6$ & 83 & 97 & 83 & 97 \\
\hline C difficile (iv) & 5M2FC positive & $5 / 6$ & $1 / 38$ & 22.2 & $2.6 E-6$ & 83 & 97 & 83 & 97 \\
\hline C difficile (v) & $\begin{array}{l}3 \mathrm{MI} \text { negative, } \\
5 \mathrm{M} 2 \mathrm{FC} \text { positive }\end{array}$ & $4 / 6$ & $0 / 38$ & 20.4 & $6.3 E-6$ & 67 & 100 & 100 & 95 \\
\hline Campylobacter & $\begin{array}{l}\text { No terpenes/ } \\
\text { hydrocarbons }\end{array}$ & $5 / 5$ & $3 / 39$ & 19.6 & $1 E-5$ & 100 & 92 & 63 & 100 \\
\hline Rotavirus & EDD positive & $5 / 5$ & $1 / 39$ & 27.9 & $1.3 E-7$ & 100 & 97 & 83 & 100 \\
\hline Non-rota enteritis & $\begin{array}{l}\text { Ammonia positive, } \\
\text { EDD negative }\end{array}$ & $11 / 19$ & $1 / 25$ & 15.4 & $8.6 E-5$ & 63 & 96 & 92 & 77 \\
\hline
\end{tabular}

The strength of association is shown using the $\chi^{2}$ test with Yates' correction, sensitivity and specificity, and positive predictive (PPV) and negative predictive (NPV) values.

3MI, 3-methylindole; 2FC, 2-furancarboxaldehyde; 5M2FC, 5-methyl-2-furancarboxaldehyde; EDD, ethyl dodecanoate. 
approach is not widely used and unlike our approach requires the organism to be cultured before it can be identified.

Board spectrum antibiotics suppress the growth of $E$ coli while permitting the overgrowth of $C$ difficile. Thus the reduced production of methylindole in patients with this infection is likely to be a result of the disruption of metabolism by $E$ coli. This explanation for the profile of VOCs from stool samples is supported by the fact that $C$ difficile does not produce indole from tryptophan. ${ }^{10}$ It is noteworthy that stool from patients who have received antibiotic prophylaxis during conditioning for BMT contain no 3-methylindole, consistent with suppression of $E$ coli, but there were no furans in these samples, supporting the association between $C$ difficile and furans.

Multiple enzyme immunoassays may provide a rapid test for $C$ difficile. ${ }^{11}$ ELISAs have a sensitivity of $70-90 \%$ and specificity of $99 \% .{ }^{12}$ As quick as they may be, they are not used for near patient testing and are still dependent on transport to a microbiology laboratory. This is reflected in the diagnostic delay of 8.2 days reported by Johal and colleagues. $^{4}$ Furans alone have a sensitivity (83-100\%) and specificity (79-97\%) to rival ELISAs but have the potential for near patient testing. Furans in the absence of methylindole are less sensitive (66-83\%) but are more specific (97$100 \%)$.

Ethyl dodecanoate is $100 \%$ sensitive for the diagnosis of rotavirus. However, the sensitivity of this marker for rotavirus was $97 \%$, as a result of one false positive. It is possible that the diagnosis of rotavirus was missed by electron microscopy, particularly as the patient had adenovirus and a second virus may have been overlooked. Alternatively, as diarrhoea due to rotavirus is more common in young children than in adults, ethyl dodecanoate may simply indicate stool derived from children. Ethyl dodecanoate has not been previously reported in patients with rotavirus although a relative increase in propionic and butyric acids has been reported. ${ }^{13}$ We found that while short chain (C3-C5) fatty acids were most abundant in patients with rotavirus, there were also significant amounts in patients with Campylobacter and $C$ difficile, so they have no diagnostic value.

Enteritis due to giardiasis and viruses other than rotavirus can be diagnosed with $100 \%$ specificity, albeit with a sensitivity of $61 \%$, if ammonia is present and ethyl dodecanoate absent, in the VOCs from a stool sample from a patient with diarrhoea. No such marker of viral enteritis has been described previously.

The findings from patients with Campylobacter infection were surprising. At present, we conclude that terpenes and hydrocarbons are not present in the VOCs from such patients.

It remains to be seen whether this approach can be applied to non-infectious gastrointestinal disorders; this is an area of active exploration.

The results we have described may be method dependent. Changing the SPME fibre and the protocol for GC-MS may reveal other pertinent volatile markers which are even more sensitive and specific. We believe there is significant scope for improving the speed of the vapour analyses.

We have shown that stool samples from patients with infectious diarrhoea contain characteristic chemical fingerprints that may be used to make a diagnosis without the need for complex manipulation such as culture, ELISA, or electron microscopy. In remains to be seen whether other gastrointestinal ailments can be diagnosed by this method. The use of gas from a simple headspace device should reduce the risk of cross infection. The development of an inexpensive device capable of near patient testing will hasten diagnosis, saving time and money.

\section{ACKNOWLEDGEMENTS}

We would like to thank K Garner, University of the West of England, Bristol, for assistance in the GC-MS experiments. This work was funded by an award from the United Bristol Hospitals' Charitable Trust.

\section{Authors' affiliations}

C S J Probert, Department of Medicine, Bristol Royal Infirmary, Bristol, UK

P R H Jones, N M Ratcliffe, Faculty of Applied Sciences, Centre for Research in Analytical, Materials, and Sensor Sciences, University of the West of England, Coldharbour Lane, Bristol BS16 1QY, UK

\section{REFERENCES}

1 Spencer RC. Clinical impact and associated costs of Clostridium difficileassociated disease. J Antimicrob Chemothe 1998;41(suppl):5-12.

2 McFarland LV, Nulligan ME, Kwol RY, et al. Nosocomial acquisition of Clostridium difficile infection. N Engl J Med 1989;26:204-10.

3 Lew JF, Glass RI, Petric M, et al. 6-year retrospective surveillance of gastroenteritis viruses at 10 emergency medical centres in United States and Canada. Pediatr Infect Dis J 1990;9:709-14.

4 Johal SS, Hammond J, Wight N, et al. Role of flexible sigmoidoscopy in C difficile-associated diarrhoea (CDAD). Gut 2002;51(suppl 3):G414.

5 De Lacy Costello BPJ, Ewen RJ, Gunson HE, et al. The development of a sensor system for the early detection of soft rot in stored potato tubers. Meas $\mathrm{Sc}$ Technol 2000; 11:1685-91.

6 De Lacy Costello BPJ, Evans P, Ewen RJ, et al. GC-MS analyses of volatile organic compounds from potato tubers inoculated with Phytophthora infestans or Fusarium coeruleum. Plant Pathol 2001;50:489-96.

7 Vracko R, Sherris J C. Indole-spot-test in bacteriology. Am J Clin Pathol 1963;39:429-32

8 Johnson LL, McFarland LV, Dearing P, et al. Identification of Clostridium difficile in stool specimens by culture-enhanced gas-liquid chromatography J Clin Microbiol 1989;27:2218-221.

9 Nonhoff C, Struelens MJ, Serruys E. Evaluation of gas-liquid chromatography (GLC) for rapid detection of Clostridium difficile in fecal specimens. Acta Clin Belg 1995; 50:76-80.

10 Koneman EW, Allen SD, Janda WM, et al, eds. Colour atlas and textbook of diagnostic microbiology, 5th edn. Philadelphia: Lippincott, 1997.

11 Delmée M. Laboratory diagnosis of Clostridium difficile disease. Clin Microbiol Infect 2001;7:411-37.

12 Gerding D N, Johnson S, Peterson LR, et al. Clostridium difficile-associated diarrhoea and colitis. Infect Control Hosp Epidemiol 1995; 16:459-77.

13 Brooks JB. Review of frequency-pulsed electron-capture gas-liquid chromatography studies of diarrheal disease caused by members of the family Enterobacteriaceae, Clostridium difficile and Rotavirus. J Clin Micriobiol 1986;24:687-91. 